\title{
Accuracy and Precision in Quantitative Fluorescence Microscopy
}

\section{Citation}

Waters, Jennifer C. 2009. Accuracy and precision in quantitative fluorescence microscopy. The Journal of Cell Biology 185(7): 1135-1148.

\section{Published Version}

doi:10.1083/jcb.200903097

\section{Permanent link}

http://nrs.harvard.edu/urn-3:HUL.InstRepos:4875892

\section{Terms of Use}

This article was downloaded from Harvard University's DASH repository, and is made available under the terms and conditions applicable to Other Posted Material, as set forth at http:// nrs.harvard.edu/urn-3:HUL.InstRepos:dash.current.terms-of-use\#LAA

\section{Share Your Story}

The Harvard community has made this article openly available.

Please share how this access benefits you. Submit a story.

Accessibility 


\title{
Accuracy and precision in quantitative fluorescence microscopy
}

\author{
Jennifer C. Waters \\ Harvard Medical School, Department of Cell Biology, Boston, MA 02115
}

The light microscope has long been used to document the localization of fluorescent molecules in cell biology research. With advances in digital cameras and the discovery and development of genetically encoded fluorophores, there has been a huge increase in the use of fluorescence microscopy to quantify spatial and temporal measurements of fluorescent molecules in biological specimens. Whether simply comparing the relative intensities of two fluorescent specimens, or using advanced techniques like Förster resonance energy transfer (FRET) or fluorescence recovery after photobleaching (FRAP), quantitation of fluorescence requires a thorough understanding of the limitations of and proper use of the different components of the imaging system. Here, I focus on the parameters of digital image acquisition that affect the accuracy and precision of quantitative fluorescence microscopy measurements.

\section{What information is present in a fluorescence microscopy digital image?}

Quantitative microscopy measurements are most often made on digital images. A digital image is created when the optical image of the specimen formed by the microscope is recorded by a detector (usually a charge-coupled device [CCD] camera [Moomaw, 2007; Spring, 2007] or photomultiplier tube [PMT; Art, 2006]) using a two-dimensional grid of equally sized pixels. The pixels spatially sample the optical image, such that each pixel represents a defined finite sized area in a specific location in the specimen.

Correspondence to jennifer_waters@hms.harvard.edu
During acquisition of the digital image, the photons that are detected at each pixel are converted to an intensity value that is correlated to, but not equal to, the number of detected photons (Pawley, 2006c). In fluorescence microscopy, the intensity value of a pixel is related to the number of fluorophores present at the corresponding area in the specimen. We can therefore use digital images to extract two types of information from fluorescence microscopy images: (1) spatial, which can be used to calculate such properties as distances, areas, and velocities; and (2) intensity, which can be used to determine the local concentration of fluorophores in a specimen.

\section{Accuracy and precision}

Every quantitative measurement contains some amount of error. Error in quantitative fluorescence microscopy measurements may be introduced by the specimen, the microscope, or the detector (Wolf et al., 2007; Joglekar et al., 2008). Error shows itself as inaccuracy and/or imprecision in measurements. Inaccuracy results in the wrong answer. For example, with an inaccurate $\mathrm{pH}$ meter one might carefully measure the $\mathrm{pH}$ of a basic solution many times, each time finding the $\mathrm{pH}$ to be 2.0. Imprecision, on the other hand, results in variance in repeated measurements and therefore uncertainty in individual measurements. With an imprecise $\mathrm{pH}$ meter, repeated measurements of a solution with $\mathrm{pH} 7.0$ might have a distribution ranging from 5.0-9.0, with an average value of 7.0. Although the average value of these repeated measurements is accurate, any individual measurement may be inaccurate. The importance of accuracy is obvious. Precision is equally important in quantitative fluorescence microscopy because we are often forced to make only one measurement (for example, one time-point in a live-cell time-lapse experiment). In addition, we are usually measuring biological specimens that have some level of natural variability, so variance seen in measurements made on different cells will be caused by both biological variability and that which is introduced when making the measurement. To use a fluorescence microscope and digital detector to quantitate spatial and intensity information from biological specimens, we must understand and reduce the sources of inaccuracy and imprecision in these types of measurements.

\section{Signal, background, and noise}

In quantitative fluorescence microscopy, we want to measure the signal coming from the fluorophores used to label the object of interest in our specimen. For example, consider live cells expressing GFP-tubulin in which we wish to measure the amount of tubulin polymer. The signal we are interested in is the photons emitted from GFP bound to tubulin incorporated into microtubules. We use the pixel intensity values in the digital image to localize the tubulin polymers and make conclusions about the quantity of microtubules. However, the intensity values in the digital images of

\footnotetext{
(C) 2009 Waters This article is distributed under the terms of an Attribution-Noncommercial-Share Alike-No Mirror Sites license for the first six months after the publication date (see http://www.jcb.org/misc/terms.shtml). After six months it is available under a Creative Commons License (Attribution-Noncommercial-Share Alike 3.0 Unported license, as described at http://creativecommons .org/licenses/by-nc-sa/3.0//.
} 
Figure 1. Background fluorescence decreases precision of fluorescence intensity measure-

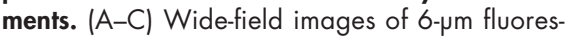
cent beads, all displayed with the same scaling so relative intensity is evident. All images were collected with the same microscope (model TE2000U; Nikon) and the same camera (ORCA. AG; Hamamatsu Photonics) using a PlanApochromat 60x 1.4 NA oil objective lens (Nikon) and MetaMorph software. (A) Fluorescent beads (mounted in PBS) with minimal background fluorescence. A 400-ms exposure time was used, and the maximum intensity value of the beads is $\sim 3,800$. Bar $=5 \mu \mathrm{m}$. (B) A solution of fluorophore (with the same spectral characteristics as the fluorophore in the bead, diluted in PBS) was added to the specimen to increase the background fluorescence. The exposure time had to be decreased to $100 \mathrm{~ms}$ to get the same maximum intensity value of the beads, $\sim 3,800$. (C) Image $B$, after background subtraction. Because a shorter exposure time was used in $B$, fewer
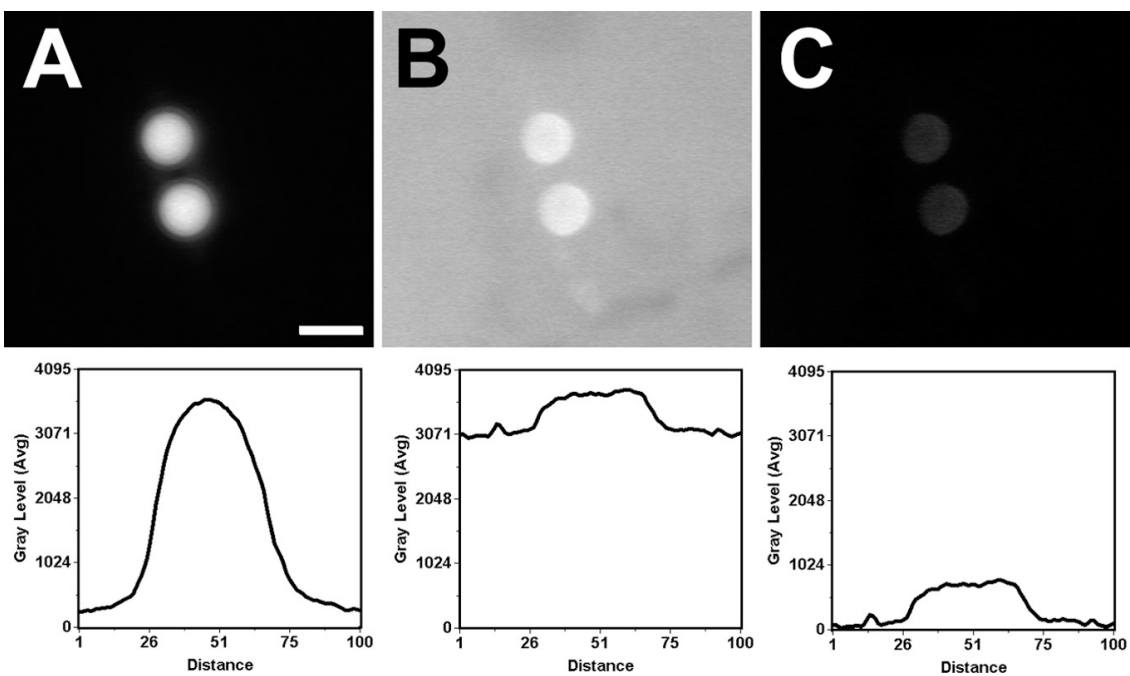
photons from the beads were collected than in $A$. Collecting fewer photons from the object of interest means a higher contribution of Poisson noise, and less precise quantitation of fluorescence intensity values. Therefore, one should work to remove background fluorescence from the image (see Table I) before background subtraction.

the microtubules represent not only the signal of interest coming from the microtubules, but also background and noise (Swedlow et al., 2002; Murray et al., 2007; Wolf et al., 2007).

Background adds to the signal of interest, such that the intensity values in the digital image are equal to the signal plus the background(Fig. 1). Background in a digital image of a fluorescent specimen comes from a variety of sources. In our example, monomers of tubulin that remain in the cytoplasm contribute to the background, as does the cell culture medium the specimen is mounted in which contains phenol red, vitamins, and other components that fluoresce. To accurately and precisely measure the signal of interest, background should be reduced as much as possible, and must be subtracted from measurements (Fig. 1; more on this later).

Noise causes variance in the intensity values above and below the "real" intensity value of the signal plus the background. The extent of deviation differs from one pixel to the next in a single digital image, with the maximum variance in an image referred to as the noise level (Fig. 2 A). Noise causes imprecision in measurements of pixel intensity values, and therefore a level of uncertainty in the accuracy of the measurements. To detect the presence of a signal, the signal must be significantly higher than the noise level of the digital image
(Fig. 2, B and C). If the signal is at or below the noise level, the variation in intensity caused by noise will make the signal indistinguishable from the noise in quantitative measurements (Fig. 2 B). As the signal increases relative to the noise level, measurements of the signal become increasingly more precise (Fig. 2 C). The precision of quantitative microscopy measurements is therefore limited by the signal-to-noise ratio (SNR) of the digital image. SNR affects spatial measurements as well as intensity measurements; precise determination of the location of a fluorescently labeled object depends on SNR (Fig. 2, D and E) (Churchman et al., 2005; Yildiz and Selvin, 2005; Huang et al., 2008; Manley et al., 2008).

Poisson noise. One type of noise found in fluorescence microscopy digital images comes from the signal we are trying to measure. Measurements of stochastic quantum events, such as numbers of photons, are fundamentally limited by Poisson statistics (Pawley, 1994, 2006a). This means that the number of photons counted in repeated measurements of an ideal, unchanging specimen will have a Poisson distribution. The number of photons counted in a single measurement therefore has an intrinsic statistical uncertainty called Poisson noise (also referred to as shot noise, signal noise, or photon noise). The maximum variance in the number of counted photons that can be attributed to Poisson noise is determined by the Poisson distribution and is equivalent to the square root of the total number of detected photons. This formula applies to the number of detected photons, not the arbitrary intensity values reported by detectors. Detected photons, $p$, can be calculated from intensity values using the equation

$$
p=\left[\left(f /\left(i_{\max }-o\right)\right) \times(i-o)\right],
$$

where $f$ is the full well capacity of the detector, $i_{\max }$ is the maximum intensity value the detector can produce, $i$ is the intensity value being converted to photons, and $o$ is the detector offset. The detector values can be obtained from the technical specification sheets available on the detector manufacturer's website.

Poisson noise cannot be reduced or eliminated. However, as the number of counted signal photons increases, the Poisson noise becomes a smaller percentage of the signal and the SNR increases. Working to increase the number of signal photons collected will therefore increase the accuracy and precision of quantitative measurements.

Maximizing signal. The intensity of the signal in digital fluorescence microscopy images is affected by every step along the path to quantitation, including specimen preparation, the microscope, and the detector (Table I, Table II). 

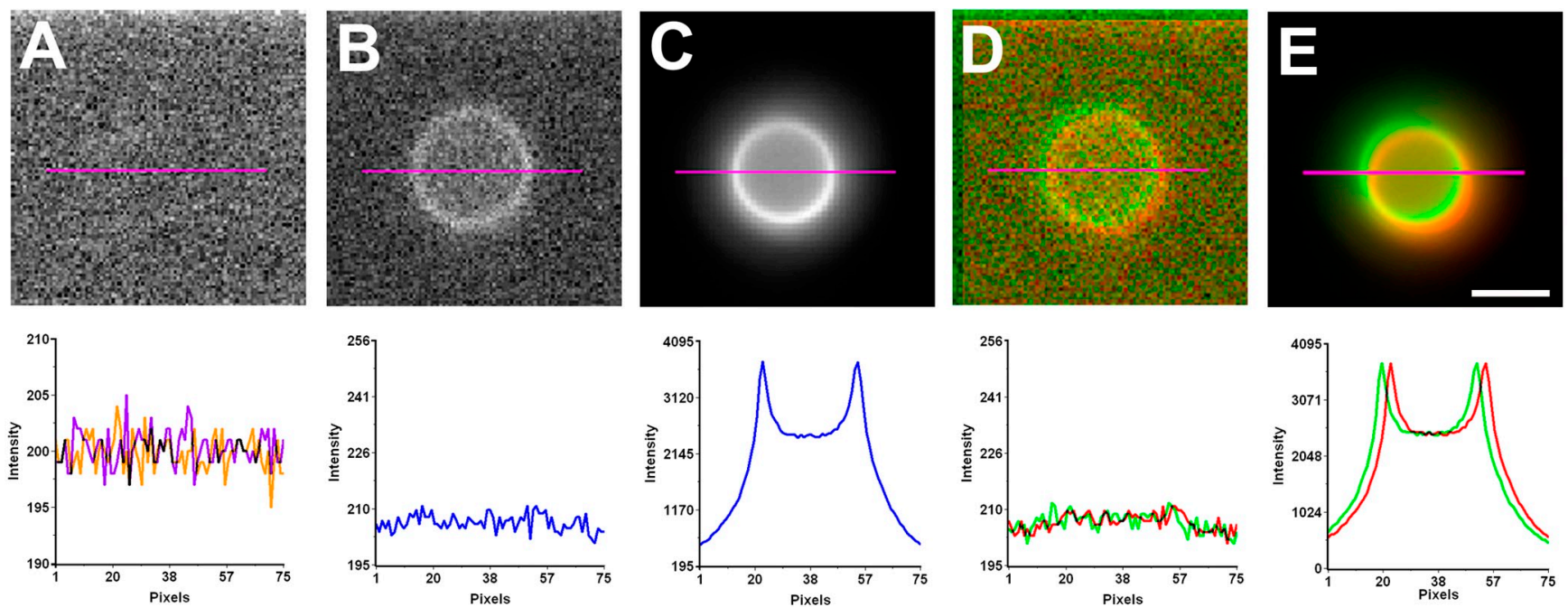

Figure 2. The importance of SNR in intensity and spatial measurements. (A) A digital image taken with a cooled CCD camera (ORCA-AG; Hamamatsu Photonics), with no light sent to the camera. Using MetaMorph software, a line (shown in red) was drawn across the bead and a line-scan graph was generated to show the intensity value of the pixels along the line. The graph shows line-scans of two similar images, taken in quick succession. The intensity values in the images fluctuate (range, 195-205) around the camera digital offset value of 200. Notice that the fluctuation in intensity values changes at each pixel from one image to the next. This variance is due primarily to thermal and readout noise from the CCD camera, and the extent of the variance will differ depending on the camera. This type of noise is superimposed on every fluorescence microscopy image. (B-E) Images of 6 - $\mu \mathrm{m}$ beads that are fluorescently stained along their perimeter were collected with a wide-field microscope (model TE2000U; Nikon) using a Plan-Apochromat 60x 1.4 NA oil objective lens, the same camera as in A (ORCA-AG; Hamamatsu Photonics), and MetaMorph software. Line-scans generated as described for A. (B) An image of the bead taken with a 100-ms exposure time. The SNR is very low, making the bead indistinguishable from the noise in the line scan. (C) An image of the same bead as in B, taken with a longer (3 s) exposure time. The high SNR of this image would make quantitation of the intensity of the bead, or localization of the edge of the bead, highly precise. (D and E) The same bead images in B and C. Two images of the bead were taken, and one copy pseudo-colored red and one copy pseudo-colored green. The pseudo-colored images were shifted relative to one another by a few pixels and merged. (D) With low SNR images, it is nearly impossible to precisely locate the edges of the beads. (E) With high SNR images, the intensity line scan can be fit to Gaussian curves and the center located with nanometer precision. This allows the distance between objects of two wavelengths to be precisely determined, even if it is well below the resolution limit of the microscope (Churchman et al., 2005; Yildiz and Selvin, 2005; Huang et al., 2008; Manley et al., 2008). Bar = $5 \mu \mathrm{m}$.

The specimen. Fluorophores vary greatly in their intrinsic brightness and the rate at which they photobleach; an easy way to maximize signal is to choose a brighter and more photo-stable fluorophore (Diaspro et al., 2006; Tsien et al., 2006). The brightness of a fluorophore is determined primarily by its extinction coefficient and quantum yield, properties that are dependent on the fluorophore's environment (Diaspro et al., 2006). It should be noted that new fluorescent proteins are routinely introduced that outperform their predecessors; it is therefore advisable to search the current scientific literature for the latest variants.

Fixed specimens should be mounted in a glycerol-based mounting medium (Egner and Hell, 2006; Goodwin, 2007) that contains an anti-photobleaching inhibitor (Diaspro et al., 2006). No one antiphotobleaching reagent is the best, as each reagent is more or less effective for a given fluorophore (Diaspro et al., 2006). Review the fluorophore manufacturer's product information or the relevant scientific literature (Shaner et al., 2005;
Giepmans et al., 2006; Johnson, 2006) to make the best choice of fluorophore and anti-photobleaching reagent for your specimen and experiment. Goodwin (2007) provides a complete discussion of the importance of mounting medium choice to both signal intensity and resolution.

The microscope. To get the brightest signal while minimizing specimen damage, it is important to use illumination wavelengths that will optimally excite the fluorophore and to collect as many of the emission photons as possible (Ploem, 1999; Rietdorf and Stelzer, 2006). Fluorescence spectra that show the absorption and emission efficiency of fluorophores are available from the manufacturer or in the scientific literature (for example, see Shaner et al., 2005), and filter manufacturers provide spectra online that show the percent transmission of their filters across wavelength. It is important to compare the spectra for the fluorophore you are imaging to spectra for the fluorescence filter sets (and/or laser illumination line) to ensure you are using the correct wavelengths of light to excite the fluorophore and collecting as much of the emission light as possible (Ploem, 1999; Rietdorf and Stelzer, 2006). There are several useful online tools available for matching fluorophores to filters (for example, as of the date of this publication Invitrogen has a very useful tool on their website: http://www.invitrogen .com/site/us/en/home/support/ResearchTools/Fluorescence-SpectraViewer.html).

In an epifluorescence microscope, the objective lens both illuminates the specimen and collects photons emitted from fluorophores to form the optical image. The numerical aperture (NA) of the objective lens (marked on the barrel of the lens after the magnification; Keller, 2006) is an important determinant of the brightness of the optical image. The number of photons an objective can collect from a specimen (and therefore the brightness of the image) increases with $\mathrm{NA}^{2}$. Brightness of an objective is also determined by properties such as transmission and correction for aberration (Keller, 2006). Spherical aberration caused by the objective lens (Hell and Stelzer, 1995; 


\section{Increase signal:}

$\checkmark$ Choose a bright (high quantum yield, high extinction coefficient) and photo-stable fluorophore ${ }^{a}$

$\checkmark$ Image through a clean No. 1.5 coverslip $^{b}$

$\checkmark$ Mount specimen as close to the coverslip as possible

$\checkmark$ Use high NA clean objective lens with lowest acceptable magnification ${ }^{d}$

$\checkmark$ Choose fluorescence filter sets that match fluorophore spectra ${ }^{d}$

$\checkmark$ Align arc lamp for Koehler illumination ${ }^{d}$

$\checkmark$ For fixed specimens, use a glycerol-based mounting medium containing anti-photobleaching inhibitors ${ }^{3}$

$\checkmark$ Remove DIC Wollaston prism and analyzer from light pathe

$\checkmark$ Use a cooled CCD camera with at least $60 \%$ quantum efficiency ${ }^{d}$

$\checkmark$ Use camera binning ${ }^{d}$

\section{Decrease noise:}

$\checkmark$ Use a cooled CCD camera with less than 8 electrons readout noise and negligible dark noise ${ }^{f}$

$\checkmark$ Use amplification (e.g., EM-CCDs) only when signal is limiting ${ }^{f}$

$\checkmark$ Increase signal (see above) to reduce relative contribution of Poisson noise ${ }^{f}$

\section{Decrease background:}

$\checkmark$ Clean coverslips and optics ${ }^{e}$

$\checkmark$ Perfect fluorophore labeling protocol to minimize nonspecific labeling ${ }^{9}$

$\checkmark$ Mount specimens in minimally fluorescent medium (e.g., without phenol red) ${ }^{d}$

$\checkmark$ Use band-pass filter sets that block autofluorescence ${ }^{d}$

$\checkmark$ Turn off the room lights ${ }^{d}$

$\checkmark$ Close down the field diaphragm to illuminate only the object of interest ${ }^{d}$

$\checkmark$ When out-of-focus fluorescence is high, consider using deconvolution, confocal, or TIRF ${ }^{\mathrm{h}}$

a(Diaspro et al., 2006)

b(Keller, 2006)

'(Goodwin, 2007)

d'Waters, 2007)

e(Inové and Spring, 1997)

f(Moomaw, 2007)

${ }^{9}($ Allan, 2000)

h(Murray, 1998)

Goodwin, 2007) or introduced by the specimen (Egner and Hell, 2006) decreases image intensity (North, 2006; Waters, 2007; Waters and Swedlow, 2007). Spherical aberration occurs when there is a relatively large difference in refractive index between the specimen and the lens immersion medium; for example, when an oil immersion lens is used to image a specimen in an aqueous solution such as cell culture medium (Egner and Hell, 2006). Spherical aberration caused by refractive index mismatch generally increases with distance from the coverslip (Joglekar et al., 2008). Spherical aberration can be addressed using water immersion objective lenses (Keller, 2006), by using an objective lens with a correction collar (Keller, 2006; Waters, 2007), or by immersion oil refractive index matching (Goodwin, 2007). For fixed specimens, spherical aberration is reduced by mounting fixed specimens in a mounting medium with a refractive index similar to that of the immersion medium (e.g., mounting medium with a high concentration of glycerol will have a refractive index close to that of standard immersion oil).

The detector. The number of photons reaching the detector that are collected and contribute to the intensity values in a digital image depends on the quantum efficiency (QE) of the detector, and how long the signal is allowed to integrate on the detector (usually referred to as the exposure time). QE is a measure of the percentage of photons reaching the detector that are counted (Moomaw, 2007). The QE of researchgrade CCD cameras most often used for quantitation of fluorescence images ranges from $60 \%$ to over $90 \%$, whereas the QE of PMTs used in point-scanning confocals is much lower, usually 10 $20 \%$ (although the effective QE is significantly less; see Pawley, 2006b). QE values are available online from the detector manufacturer.
Increasing the exposure time allows the flux of photons coming from the specimen to accumulate (as electrons) in the detector, increasing the intensity values in the image-up to a point (Moomaw, 2007; Spring, 2007; Waters, 2007). Detectors have a limited capacity to hold electrons; if this capacity is reached, the corresponding pixel will be "saturated" and any photons reaching the detector after saturation will not be counted. The linearity of the detector is therefore lost, and saturated images cannot be used for quantitation of fluorescence intensity values. Choosing to "crop out" saturated areas is not acceptable (unless they can be shown to be irrelevant to the experimental hypothesis) because it will select for the weaker intensity parts of the specimen. Saturation should be avoided by using image acquisition software to monitor intensity values when setting up the acquisition parameters (Table II). 


\section{Acquire optical images}

- Set up specimen and imaging system for optimal signal detection, low background, and low noise (Table I)

2. Acquire digital images

- Use software to monitor intensity values in the image to choose the best acquisition settings ${ }^{a}$

- Use full dynamic range of the camera for fixed specimens ${ }^{a}$

- For live-cell work, it is often necessary to sacrifice SNR to minimize specimen exposure to light and maintain cell health and viability

- Consider binning to increase SNR

- Avoid high camera gain when a large dynamic range is needed ${ }^{a}$

- Avoid saturating pixels in the image

- Eliminate or minimize exposure of specimen to fluorescence excitation light prior to image acquisition ${ }^{a}$

- Focus carefully, preferably with phase or $\mathrm{DIC}^{\mathrm{b}}$

\section{Store images}

- Always save the raw images

- Use either no compression or lossless compression ${ }^{c}$

4. Process images

- Use flat-field correction to correct for uneven illumination ${ }^{d}$

- Be sure any other image processing used prior to quantitation preserves relative intensity values,

\section{Analyze images}

- Subtract local background value from intensity measurements ${ }^{\mathrm{e}}$

- Do not measure intensity values on compressed or pseudo-colored images ${ }^{c}$

- Validate image segmentation and analysis method ${ }^{f}$

- Calculate and report the error in your measurements ${ }^{d, g}$

a(Waters, 2007)

b(Inové and Spring, 1997)

'(Russ, 2007)

d(Wolf et al., 2007)

e(Hoffman et al., 2001)

(Dorn et al., 2008)

g/Cumming et al., 2007)

In most live biological specimens, saturation is much less of a problem than collecting enough signal to get adequate SNR images for quantitation. Many research-grade cooled cameras allow binning of adjacent pixels on the CCD chip. With all other acquisition parameters being equal, binning on the CCD chip increases the intensity of the pixels without increasing readout noise, resulting in a higher SNR digital image (Moomaw, 2007; Spring, 2007; Waters, 2007). However, because the resulting pixels represent a larger area of the specimen (i.e., $4 x$ larger with a $2 \times 2$ bin), binning decreases the resolution of the digital image (Fig. 3). In many low-light imaging experiments, however, the decrease in resolution is well worth the increase in SNR (Table II).

Background fluorescence reduces dynamic range and decreases SNR. Although it's true that background fluorescence can and must be subtracted from quantitative measure- ments of intensity, it is also very important to first reduce background as much as possible (Fig. 1, Table I). Background in an image effectively reduces both the dynamic range and the SNR. Dynamic range of a CCD camera is defined as the full well capacity of the photodiodes (i.e., the number of photons that can be detected per pixel before saturation) divided by the detector noise (Moomaw, 2007; Spring, 2007). High dynamic range is particularly important for collecting an adequate number of signal photons from both dim and bright areas of the specimen. Photons from background sources fill the detector, limiting the number of signal photons that can be collected before the detector saturates (Fig. 1) and effectively decreasing dynamic range. In addition, recall that the number of photons counted defines the Poisson noise level in an image. Poisson noise is equal to the square root of the signal photons plus background photons; higher background therefore means higher
Poisson noise. Subtracting a constant background value from intensity measurements does not change the variance due to Poisson noise; the presence of background therefore reduces image SNR.

A common source of background in biological specimens is out-of-focus fluorescence. In fluorescence microscopy, the illuminating light is focused at the image focal plane by the objective lens, such that maximum excitation of fluorophores occurs at the focal plane (Hiraoka et al., 1990). However, illuminating light above and below the image focal plane excites fluorophores above and below the image focal plane. Light emitted from these out-of-focus fluorophores is collected by the objective lens, and appears as out-of-focus background in the in-focus image of the specimen. In wide-field epifluorescence microscopy, adjusting the diameter of the field diaphragm to match the visible field of view minimizes the illumination of out-of-focus 
Figure 3. Resolution and sampling. $(A-C) I m-$ ages of the same pair of 150-nm green fluorescent beads collected with a microscope (model TE2000U; Nikon), a Plan-Apochromat 100x 1.4 NA oil objective lens, and MetaMorph software. A camera with 6.45- $\mathrm{mm}$ photodiodes (ORCA-AG; Hamamatsu Photonics) was used, and different camera binning settings were used to vary the area of the specimen covered by one pixel. Exposure times were adjusted to reach a maximum intensity value of $\sim 3,600$ for each image. Using the equation for lateral resolution, we can calculate that the diameter of the first

minimum of the airy disk, and therefore the diameter of the bead in the optical image, should be equal to $\sim 465 \mathrm{~nm}$. Bar $=0.5 \mu \mathrm{m}$. (A) An image collected with no camera binning, and an exposure time of $200 \mathrm{~ms}$. Each pixel corresponds to $\sim 65 \mathrm{~nm}$ of the specimen, and each bead is sampled with $\sim 7$ pixels. (B) An image collected using $2 \times 2$ camera binning, and an exposure time of $50 \mathrm{~ms}$. Each pixel corresponds to $\sim 129 \mathrm{~nm}$ of the specimen, and each bead is sampled with about 3.5 pixels. (C) An image collected using $4 \times 4$ camera binning, and an exposure time of 25 ms. Each pixel corresponds to $\sim 258 \mathrm{~nm}$ of the specimen, and each bead is sampled with less than 2 pixels. The optical image is under-sampled, and the two beads can no longer be distinguished as separate from one another.

fluorophores (Hiraoka et al., 1990) and reduces background (Waters, 2007).

There are several microscopy techniques that serve to reduce the amount of out-of-focus fluorescence in the image. Confocal microscopes illuminate the specimen with a focused light source, while one or more corresponding pinholes at the image plane block out-of-focus fluorescence from reaching the detector (Pawley, 2006b). Spot-scanning confocals scan the specimen point-by-point with a single focused laser beam, whereas multi-point or slit-scanning confocals (including spinning disk confocals) use multiple pinholes or slits to illuminate the specimen more quickly (Adams et al., 2003; Tommre and Pawley, 2006). Multi-photon microscopes illuminate the specimen with a focused high-power long wavelength laser, which results in excitation of the fluorophores through absorption of multiple photons at the same time only at the focal plane (Rocheleau and Piston, 2003). In total internal reflection (TIRF) microscopy, fluorophores are excited with the evanescent wave of energy that forms when total internal reflection occurs at the boundary between media of different refractive indexes, usually the coverslip and the specimen (Axelrod et al., 1983). Deconvolution algorithms can also be used to reduce the out-of-focus fluorescence in digital images post-acquisition (Wallace et al., 2001).

Because out-of-focus fluorescence is a source of background, and background reduces SNR and dynamic range, shouldn't we always use one of the imaging methods that reduces out-of-focus fluorescence? The answer is not that simple. Each of the
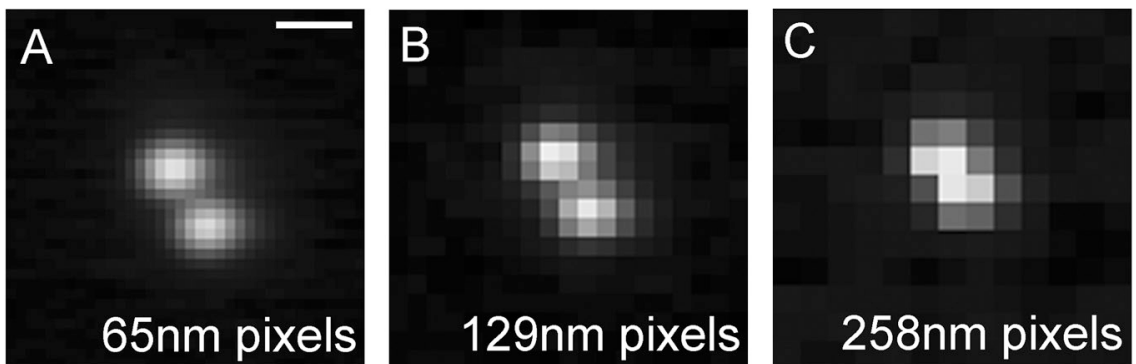

methods used to remove out-of-focus fluorescence has limitations, and may contribute additional noise to the image (Murray et al., 2007). In specimens with low levels of out-of-focus fluorescence (which is often the case in adherent cultured cells), standard wide-field fluorescence microscopy may result in the highest SNR image (Murray et al., 2007). Therefore, none of the different modes of microscopy is "better" than the other, only more or less appropriate for a particular specimen or application (Swedlow et al., 2002; Murray et al., 2007). When possible, empirical comparison of available modes is the most reliable way to ensure you are using the best imaging system for your application.

Any background that remains in a fluorescence microscopy digital image must be subtracted from intensity value measurements to reveal the signal (Table II). Consider two specimens, one with an average intensity value of 2,000 and the second with an average intensity value of 2,500. Without considering background, one might conclude that the fluorescence signal in these two specimens differs by $25 \%$. However, if the background in each image measures 1,900 , the difference is actually sixfold! Background should be subtracted following the equation

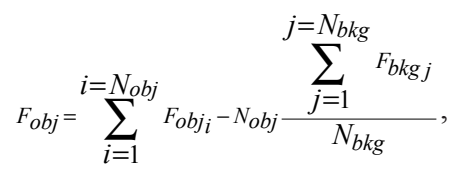

where $F$ is the fluorescence intensity measured at each pixel $i$ (pixels in the object) or $j$ (pixels in the background), $o b j$ is the object of interest, $b k g$ is the background, and $N$ is the number of pixels in the object of interest or the background. This equation corrects for different-sized regions of interest used to measure the object of interest and the background by calculating the background per pixel. This can also be achieved by using image analysis software to calculate the mean intensity value in a region of interest, as long as the number of pixels in the region of interest and the range of intensity values in those pixels are sufficient to give a precise mean (Cumming et al., 2007). To avoid errors due to an inhomogeneous background, it is best to make background measurements using pixels that are immediately adjacent to or surrounding the object of interest (for examples, see Hoffman et al., 2001 or Murray et al., 2007). This is especially important when making measurements of intracellular structures because the background in the cytoplasm is often different than the background outside of cells, and is usually inhomogeneous.

Detector noise. Fluorescence microscopy digital images are degraded by Poisson noise and by noise from the detector (Pawley, 1994, 2006a; Moomaw, 2007; Spring, 2007). Thermal noise is caused by the stochastic generation of thermal electrons within the detector, and is largely eliminated by cooling (hence the use of cooled CCD cameras; Table I). Read noise is generated by the amplifier circuitry used to measure the voltage at each pixel, and is usually the dominant source of noise in standard cooled CCD cameras designed for quantitative imaging. 
Read noise is usually expressed in the manufacturer's technical specifications as a number of electrons, meaning that the measured voltage will have a variance equal to that number of electrons (i.e., the lower the value, the lower the noise). Detectors that use signal amplification (e.g., PMTs and electron multiplying [EM] CCDs) introduce additional noise during the amplification process. For example, EM-CCD cameras amplify signal differences sufficiently to reveal clock-induced charging - stochastic variations in the transfer of charge from one pixel to another during read operations (Robbins and Hadwen, 2003; Moomaw, 2007). When possible, collecting more photons from the specimen to increase the signal (see Table I) will result in a higher SNR image than amplifying a smaller number of collected photons. The various sources of noise add as the sum of the squares:

$$
N_{\text {total }}=\sqrt{N_{\text {Poisson }}^{2}+N_{\text {read }}^{2}+N_{\text {thermal }}^{2}+\ldots} .
$$

The resulting total noise in the digital image defines a minimum expected variance in the measured intensity values. Differences in measurements that lie within the expected variance due to noise cannot be attributed to the specimen. Pawley (1994) provides a thorough review of the different sources of noise in digital microscopy images.

Noise is not a constant, so it cannot be subtracted from a digital image. However, if multiple images of the same field of view are collected and averaged together ("frame averaging"), the noise will average out and the resulting mean intensity values will be closer to the "real" intensity values of the signal plus the background (Cardullo and Hinchcliffe, 2007). Frame averaging is very useful when imaging fixed specimens with a higher noise instrument like a point-scanning confocal, but is usually impractical for quantitative imaging of live fluorescent specimens that are dynamic and susceptible to phototoxicity and photobleaching. For quantitative fluorescence imaging, noise added to the digital image during acquisition should be reduced as much as possible through choice of detector and acquisition settings (Table I).

\section{Resolution}

In digital microscopy, spatial resolution is defined by both the microscope and the detector, and limits our ability to accurately and precisely locate an object and distinguish close objects as separate from one another (Inoue, 2006; Rasnik et al., 2007; Waters and Swedlow, 2007). Objects that cannot be detected in an image cannot be resolved, so spatial resolution is dependent on image SNR (Pawley, 2006c). When imaging a dynamic specimen over time, accuracy of quantitation may be further limited by temporal resolution (the rate of image acquisition; see Dorn et al., 2005; Jares-Erijman and Jovin, 2006 for an example).

Resolution in the optical image. Lateral resolution of the optical image is defined as the distance by which two objects must be separated in order to distinguish them as separate from one another, which is equal to the radius of the smallest point source in the image (defined as the first minimum of the airy disk; Inoue, 2006). Lateral resolution $(r)$ in epifluorescence microscopy is given by

$$
r=\frac{(0.61) \lambda}{N A}
$$

where $\lambda$ is the wavelength of emission light and $N A$ is the numerical aperture of the objective lens. Numerical aperture is usually marked on the objective lens barrel, just after the magnification (Keller, 2006). Resolution in the z-axis ( $z$ ) is worse than lateral resolution in the light microscope, and is given by

$$
z=\frac{2 \lambda \eta}{(N A)^{2}},
$$

where $\eta$ is the refractive index of the specimen. It is important to understand that these equations give the theoretical resolution limits of a perfect lens used to image an ideal specimen; real lenses and specimens often introduce aberrations in the image that reduce resolution (Egner and Hell, 2006; Goodwin, 2007). The best way to know the resolution limit of your imaging system is to measure it empirically (Hiraoka et al., 1990). These equations define the theoretical resolution limits in most cases; it should be noted that a handful of very talented microscopists have found it possible to surpass these limits using specialized "super-resolution" imaging techniques (for review see Evanko, 2009).

It is a common misconception among cell biologists that confocal microscopy should be used to obtain the highest resolution images. Although confocal microscopy is very effective at increasing contrast in specimens with significant out-of-focus fluorescence (Pawley, 2006b; Murray et al., 2007), the obtainable resolution of confocal microscopy is essentially the same as conventional wide-field fluorescence microscopy (Inoue, 2006). In addition, Murray et al. (2007) recently demonstrated that the photon collection efficiency and SNR of wide-field fluorescence is generally higher than confocal microscopy for specimens with limited out-of-focus fluorescence. Confocal microscopy becomes necessary and favorable for specimens with high levels of out-of-focus fluorescence because out-of-focus fluorescence adds to background fluorescence, and therefore decreases the capacity to collect the signal of interest (Fig. 1; Murray et al., 2007).

Resolution in the digital image. The optical image is sampled by a detector to create a digital image. The resolution of a digital image acquired with a CCD camera depends on the physical size of the photodiodes that make up the chip (Rasnik et al., 2007), whereas in pointscanning confocal resolution is determined by the area of the specimen that is scanned per pixel (Pawley, 2006c). The pixel size should be at least two times smaller than the resolution limit of the microscope optics, so that the smallest possible object in the image (defined as the diameter of the airy disk) will be sampled by 4 pixels (Fig. 3; Pawley, 2006c). There is a tradeoff between resolution of the digital image and signal intensity because magnification decreases image intensity (Waters, 2007) and smaller pixels generally collect fewer photons (Fig. 3). To compensate for loss of signal due to smaller pixel size, longer camera exposure times or more intense illumination may be necessary (Fig. 3 A). If the pixel size is too large, the optical image will be under-sampled and detail will be 

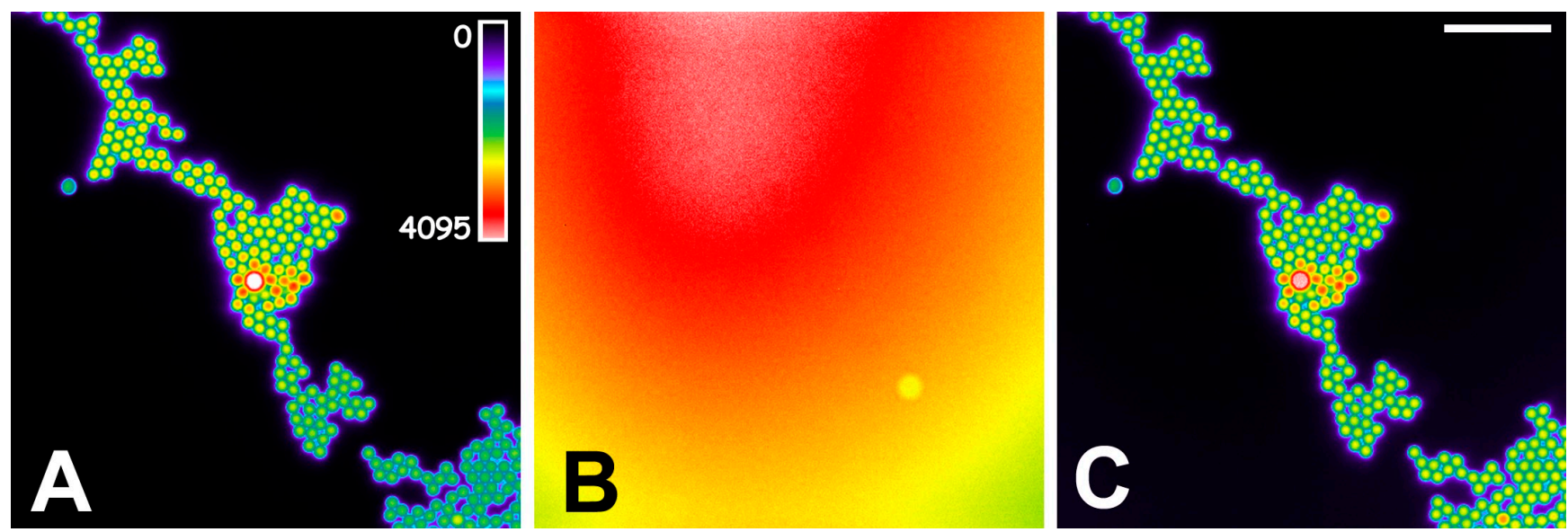

Figure 4. Non-uniform illumination results in nonuniform fluorescence. All images were collected using a microscope (model TE2000E; Nikon), a PlanApochromat 20x $0.75 \mathrm{NA}$ objective lens, a camera (ORCA-AG; Hamamatsu Photonics), and MetaMorph software. (A) An image of a field of fluorescent beads, using wide-field illumination. Individual beads contain a similar concentration of fluorophore (clumps of beads appear brighter, as is seen near the center of the image). A pseudo-color displaying the range of intensity values (see inset) was applied. Note that beads in the top left have different intensity values than the beads in the bottom right. (B) An image of a uniform field of fluorophore taken with the same microscope optics and conditions as $A$, showing uneven illumination across the field of view. This nonuniform illumination explains the nonuniform fluorescence from the beads of similar fluorophore concentration shown in A. (C) After flat-field correction (Zwier et al., 2004; Wolf et al., 2007), the image intensity values more accurately reflect the real fluorescence in the specimen. This image was obtained using the image arithmetic function in image processing software (in this case, MetaMorph) to divide the image in $\mathrm{A}$ by the image in $\mathrm{B}$. Bar $=50 \mu \mathrm{m}$.

lost in the digital image (Fig. 3 C). In livecell imaging, it is often favorable to give up some resolution (by binning pixels, for example) to increase image SNR and/or decrease photobleaching and photo damage (Waters, 2007).

Optical resolution need not limit accuracy in localization or counting. How does the resolution limit affect our ability to quantitate using fluorescence microscopy? Clearly, the size of an object that is below the resolution limit cannot be accurately measured with the light microscope. However, objects that are below the resolution can be detected and an image of the object formed by the microscope, if the imaging system is sensitive enough and the object is bright enough. Although the size of the object in the image will be inaccurate, the centroid of a high SNR image of the object can be used to locate the object with nanometer precision, far beyond the resolution limit (Churchman et al., 2005; Yildiz and Selvin, 2005; Huang et al., 2008; Manley et al., 2008).

In fluorescence microscopy, the resolution limit does not limit our ability to accurately count fluorescently labeled objects, even if the objects are below the resolution limit. If the objects are all of similar size, are all labeled with the same number of fluorophores, and the intensity of one object can be accurately determined; then intensity values can be used to count multiple objects that are too close to one another to spatially resolve. These types of measurements are very challenging to perform with accuracy, and require a thorough understanding of, and attention to, every possible pitfall (Pawley, 2000)_but they are possible. For example, the measured intensity of proteins conjugated to fluorescent proteins has been used to accurately and precisely count the number of labeled proteins localized to the kinetochore (Joglekar et al., 2006) and proteins involved in cytokinesis (Wu and Pollard, 2005).

\section{Additional threats to accuracy and precision in quantitative microscopy} Specimen preparation. Fluorescence from a fluorophore tagged to a molecule of interest is often used to measure the quantity of the tagged molecule. Fluorescent proteins expressed in live cells are excellent for quantitation; because there is a constant number of fluorophores per labeled protein, the number of photons emitted can be an accurate measure of the quantity of fluorescently labeled protein (Shaner et al., 2005; Straight, 2007; Joglekar et al., 2008). Small molecules that bind with high affinity to their target, such as calcium indicator dyes, are also reliable for quantitation (Johnson, 2006). Quantitation of live versus fixed cells is generally preferable because the fixation and extraction process can remove tagged proteins, quench the fluorescence of fluorescent proteins, and change the size and shape of cells (Allan, 2000; Straight, 2007).

One should use caution when using immunofluorescence to measure the local concentration of a protein of interest, particularly with soluble proteins. The fixation and extraction necessary to get antibodies into cells can change the quantity and localization of detectable epitopes (Melan and Sluder, 1992). Multivalent antibodies also bind with higher affinity to multiple epitopes, which can make areas with high concentration of the epitope label more efficiently than areas of low concentration (Mason and Williams, 1980). In addition, penetration of the antibody may not be consistent in different areas of the tissue, cells, and subcellular compartments (Allan, 2000). Therefore, although accurate quantitation of the emission photons from fluorescently labeled antibodies is possible, that number of photons may not accurately reflect the number of epitopes in the specimen. It is possible to use rigorous controls to demonstrate that an immunofluorescence protocol is an accurate 

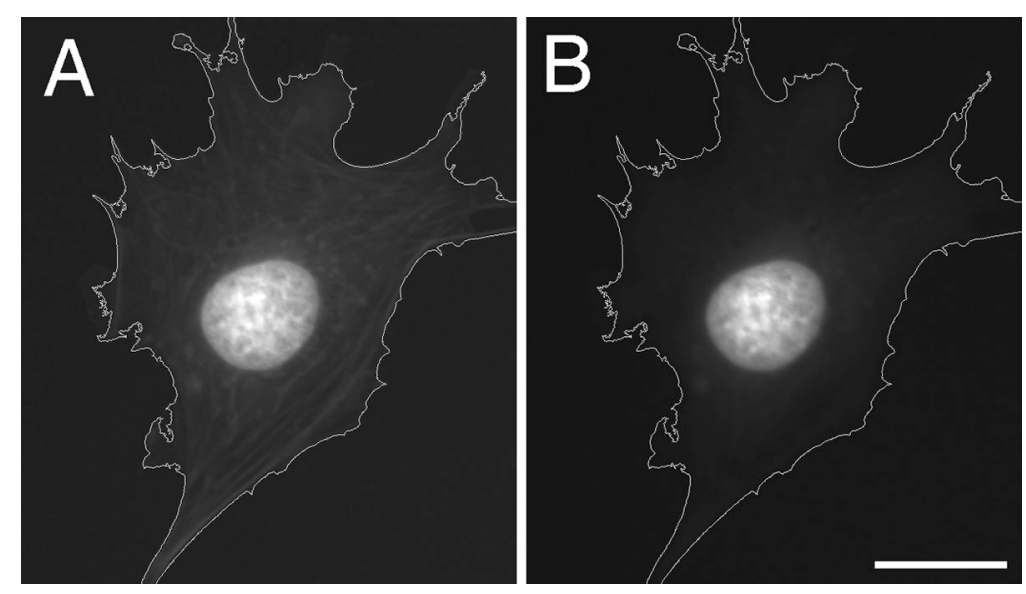

Figure 5. Bleed-through can cause inaccuracy in intensity measurements. (A and B) Images of a cell (outlined in white) labeled with DAPI (nuclei) and Bodipy-FL phalloidin (actin). Both images were collected using the same microscope (model 80i; Nikon), a Plan-Apochromat 100x NA 1.4 oil objective lens, the same camera (ORCA R2; Hamamatsu Photonics), and MetaMorph software. The same camera acquisition settings were used for both images, but they were collected using two different filters designed for imaging DAPI. (A) An image collected with a DAPI filter set containing a long-pass emission filter, which allows bleed-through of the Bodipy-FL signal in the cytoplasm. The bleed-through of the actin in the cytoplasm is just barely visible by eye in the image. The average intensity value of the cytoplasm in this image is 205. (B) An image of the same cell as in A, collected with a DAPI filter set containing a band-pass emission filter, which blocks bleed-through of the Bodipy-FL signal in the cytoplasm. The average intensity value of the cytoplasm in this image is 91 , over $50 \%$ less than the image in $\mathrm{A}$. Bar $=10 \mu \mathrm{m}$.

measure of a particular epitope of interest (Mortensen and Larsson, 2001).

Measuring fluorescence deep into specimens can also be problematic for measurements of signal intensities. In biological specimens, light scattering and optical aberrations increase with distance from the coverslip and decrease signal intensity (Murray, 2005). These effects are difficult to characterize and correct for in inhomogeneous biological samples. Although the effects of light scattering are minimized when using multi-photon illumination (Rocheleau and Piston, 2003), the accuracy of quantitation of intensity values is limited by optical aberrations and dramatically increased photobleaching in the focal plane (Patterson and Piston, 2000).

Non-uniform illumination. Fluorescence emission is generally proportional to the intensity of the illuminating light (except when fluorophore ground state depletion occurs; see Tsien et al., 2006; Murray et al., 2007). Therefore, if a uniform fluorescent sample is unevenly illuminated, the resulting fluorescence will usually be uneven as well. Uneven illumination can be extremely detrimental to quantitative measurements because it may cause the intensity of an object in one area of the field of view to measure differently than the intensity of an object of equal fluorophore concentration in another area of the field of view (Fig. 4 A). To reduce uneven illumination, the wide-field fluorescence microscope should be carefully aligned for Koehler illumination (Salmon and Canman, 2001). Scrambling the image of the light source before it enters the microscope (using a liquid light guide, for example) can increase the uniformity of illumination across the field of view (Nolte et al., 2006). In many cases, completely uniform illumination is impossible to achieve, and one must instead correct for uneven illumination before making quantitative measurements (Fig. 4; Table II; Zwier et al., 2004; Wolf et al., 2007). To perform the correction, an image of a uniform fluorescent sample is collected to reveal the uneven illumination pattern (Fig. $4 \mathrm{~B}$ ). The image to be corrected is then divided by the image of the uniform fluorescent sample (using the image arithmetic functions available in most image-processing software) to obtain the flat-field corrected image (Fig. 4 C). Because the pattern of illumination may differ from day to day, it is best to collect a new image of a uniform fluorescent sample at each imaging session. A protocol for flat-field correction is described by Wolf et al. (2007).

Bleed-through and autofluorescence. When choosing fluorescence filter sets, maximizing excitation and emission collection should be balanced with minimizing bleed-through (also called crosstalk) and autofluorescence. Bleed-through of one fluorophore's emission through the filter set of another fluorophore can occur when a specimen is labeled with multiple fluorophores whose excitation and emission spectra overlap (Fig. 5; Ploem, 1999; Bolte and Cordelieres, 2006; Rietdorf and Stelzer, 2006). Many biological specimens contain native autofluorescence of similar wavelengths to the emission of many commonly used fluorophores
(Aubin, 1979; Tsien et al., 2006). For quantitative measurements, bleedthrough and autofluorescence should be avoided when possible, and measured and subtracted from measurements when unavoidable (Rietdorf and Stelzer, 2006). Avoid bleed-through and autofluorescence by carefully choosing fluorophores and filter sets. Bleed-through between two fluorophores can be detected and measured by using the fluorophore "A" filter set and camera acquisition settings to collect an image of a control sample labeled with only fluorophore "B". Autofluorescence can be detected and measured by collecting images of a control specimen that is identical to the experimental specimen except for the addition of exogenous fluorophores.

Image registration. Images of different wavelengths emitted from a single plane of a specimen may not be coincident in the optical images (Fig. 6 A). Shifts between wavelengths in $\mathrm{X}, \mathrm{Y}$, and $\mathrm{Z}$ can be introduced by a variety of sources, including wedges in fluorescence filters (Rietdorf and Stelzer, 2006) and chromatic aberrations in the objective lens (Keller, 2006). If registration between images of different wavelengths is important to your quantitative analysis, you should check for shifts between wavelengths in your microscope using submicron beads infused with multiple fluorophores (such as Tetraspeck beads, Invitrogen; protocol in Wolf et al., 2007). Consistent shifts between wavelengths can then be corrected using image-processing software (Fig. 6 B) or (for axial shifts) by using a focus motor to adjust focus between 

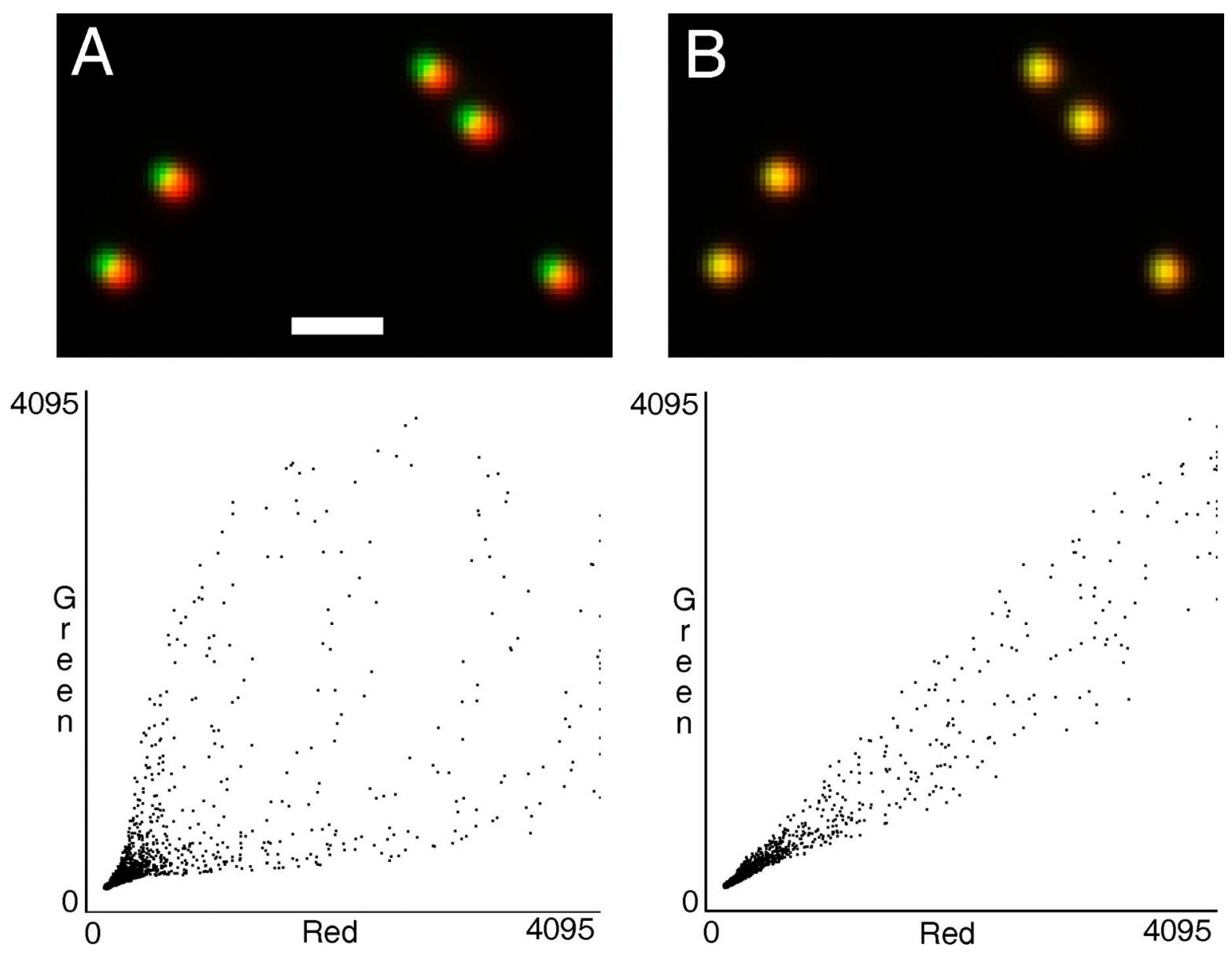

Figure 6. Shifts in image registration can affect colocalization results. (A and B) Images of 100-nm Tetra-Speck beads (Invitrogen; mounted in glycerol) that fluoresce multiple colors including red and green, collected with a microscope (model 80i; Nikon) and camera (ORCA R2; Hamamatsu Photonics) using a Plan-Apochromat 100x NA 1.4 oil objective lens and MetaMorph software. One image of the beads was collected using a filter set for green fluorescence (FITC) and a second image of the beads was collected using a filter set for red fluorescence (TRITC); all other microscope optics were the same between the two images. The two images were pseudo-colored and merged using MetaMorph software. The scatter plots (generated in MetaMorph) display the correlation between the intensity values of the red and green pixels in the images. (A) The merged image, showing a registration shift of several pixels between the red and green images. 10 sets of images were collected to determine that the shift is repeatable, and therefore most likely caused by the filter sets (not depicted). The correlation coefficient for these red and green images is only 0.72 , even though the red and green images represent the exact same beads. Bar $=1 \mu \mathrm{m}$. (B) The same images as in A, after correction for the shift in registration using MetaMorph image processing software. The correlation coefficient increased to 0.97 after the correction.

wavelengths (see Murray et al., 2007 for an example).

Focus. Focus is critical to accurate and precise quantitation of fluorescence intensity values (Murray, 2005; Table II). The distribution of intensity values along the $\mathrm{z}$-axis of the optical image depends on the size of the fluorescently labeled object and the point spread function of the microscope. For small objects imaged with high resolution optics, small changes in focus can have large effects on measured intensity values. Joglekar et al. (2008) describe a method of determining the error introduced by imprecise focus when measuring objects that are thinner than the diffraction limit of the optics. Measuring in $3 \mathrm{D}$ is almost always necessary for accurately determining intensity of objects larger than the diffraction limit, and when tracking the movements of objects that occur in 3D (De Mey et al., 2008).

Photobleaching. Almost all fluorophores photobleach when exposed to excitation light in the microscope, including all of the fluorescent proteins. The rate of photobleaching is specific to the fluorophore, its environment, and the intensity of the illuminating light (Diaspro et al., 2006). For some specimens and fluorophores, antiphotobleaching reagents can be added to the mounting medium to reduce the rate of photobleaching (Diaspro et al., 2006; Tsien et al., 2006). Depending on the rate of photobleaching, exciting fluorescent specimens before collecting images that will be used for quantitation may introduce error in measurements of signal intensity. Initial focusing and scanning of the specimen is best done using techniques such as phase or differential interference contrast (DIC) microscopy (Inoué and Spring, 1997) because the halogen light sources typically used for transmitted light illumination usually will not bleach fluorophores. To accurately measure fluorescence intensity in the same field of view over time, one should measure and correct for photobleaching that occurs while imaging (Rabut and Ellenberg, 2005). 
Image processing and storage. Some types of image processing and storage can change the relative intensity values in a digital image, rendering them unusable for quantitative measurements (Russ, 2007). For example, pseudo-coloring, bit-depth conversion, and some types of image compression (e.g., JPEG) can all compromise the intensity values in digital images (Table II). Before using any image-processing algorithm for a quantitative study, be sure to understand how it affects image intensity values. For example, imageprocessing software packages refer to many different types of algorithms as "deconvolution", but not all of these algorithms are appropriate for quantitation (see Wallace et al., 2001). In general, analysis of pixel intensity values should be done on raw images stored without further scaling or processing, or on images that have been corrected using methods that have been demonstrated to preserve the linear relationship between photons and image intensity values (e.g., flat-field corrected 16-bit TIFF images are a good choice for quantitation).

\section{Common types of quantitative microscopy analyses}

Quantitative measurements of spatial and intensity information in fluorescence microscopy digital images can be used to answer many different questions about biological specimens. Co-localization, FRET, and FRAP are three of the most commonly used quantitative microscopy methods in cell biology research. The best way to perform these types of experiments depends on many different aspects of the experimental design-for example, the molecule(s) being studied, the fluorophore(s), the type of specimen, the type of microscope, the method of image analysis, and the hypothesis being tested. It is therefore impossible to give a stepby-step protocol for any of these techniques that will work for every experiment. Even those who are very experienced in these techniques must empirically test the experimental design and imaging parameters for each novel experiment to find the optimal conditions for image acquisition and analysis. Each of these methods requires careful attention to the various pitfalls to accuracy and precision in quantitative microscopy measurements described throughout this review (and in Pawley, 2000; North, 2006; Wolf et al., 2007). In this section, I will address some of the additional specific issues surrounding these methods, and will refer the interested reader to more thorough treatments of each subject.

Colocalization. In its simplest and least informative form, colocalization analysis is performed by pseudo-coloring and merging two or more fluorescence images together, and looking for visual cues that the different wavelengths are present in the same pixels; for example, yellow pixels in a merged image of red and green fluorophores are often used to conclude that the two fluorophores "colocalize". These types of qualitative observations show, at best, that both fluorophores reside within the same 3D volume whose minimum size is defined by the resolution limits of the microscope. For high resolution wide-field or confocal microscopy, this volume is at least one order of magnitude larger than most large protein complexes. Quantitative statistical analyses of both the spatial distribution and the correlation between the intensities of different fluorescence channels is a much more informative way to measure colocalization (Day, 2005; Bolte and Cordelieres, 2006). Bolte and Cordelieres (2006) provide a thorough and focused discussion of colocalization analysis using fluorescence microscopy techniques.

The accuracy of colocalization analyses depends on the ability to discriminate between the different fluorophores, and on correct registration between images of the different wavelengths. Bleedthrough and auto-fluorescence is highly problematic for colocalization studies, and must be avoided or corrected for (discussed in detail above; Fig. 5; Rietdorf and Stelzer, 2006). Registration shifts between wavelengths limit the accuracy of high resolution colocalization measurements, and should be measured and corrected before colocalization analysis (Fig. 6).

FRET. Förster resonance energy transfer (FRET) is the nonradiative transfer of the energy absorbed by a fluorophore to a neighboring fluorophore. FRET can occur only when at least three conditions are met: (1) the emission spectrum of the donor fluorophore overlaps with the absorption spectrum of the acceptor fluorophore, (2) the donor and acceptor fluorophores are within $10 \mathrm{~nm}$ or less of one another, and (3) the emission dipole of the donor and the absorption dipole of the acceptor are orientated in the correct position (i.e., not perpendicular) relative to one another (Stryer, 1978; Schaufele et al., 2005). In cell biology research, FRET experiments usually involve tagging two molecules of interest with two different fluorophores that are capable of FRET. The presence or absence of FRET is then used to make conclusions about the proximity of the two molecules to which the different fluorophores are attached. There are many excellent reviews and books available on quantitative FRET microscopy (Gordon et al., 1998; Siegel et al., 2000; Jares-Erijman and Jovin, 2003, 2006; Sekar and Periasamy, 2003; Periasamy and Day, 2005; Chen et al., 2006; Vogel et al., 2006).

A warning regarding quantitative FRET microscopy is appropriate. FRET experiments are relatively easy to conceive, but infamously difficult to perform properly. Jares-Erijman and Jovin (2003) provide an overview of the many different available methods for detecting and measuring FRET using fluorescence microscopy. In cell biology research, quantitative measurements of FRET are most commonly performed using wide-field or confocal imaging to measure the intensities of steady-state absorption and emission of the donor and acceptor molecules (Stryer, 1978; Schaufele et al., 2005). These methods are plagued by problems that must be addressed, including auto-fluorescence, noise, photobleaching, and variations in fluorophore expression level. Spectral bleed-through of the fluorophores into the FRET channel artificially increases the observed FRET signal, and must always be measured and corrected for (Sekar and Periasamy, 2003; Schaufele et al., 2005; Vogel et al., 2006). Vogel et al. (2006) give an excellent review of the potential problems with accuracy in FRET measurements and analysis.

FRAP. In fluorescence recovery after photobleaching (FRAP) experiments, 


\section{Follow the journal's instructions for authors, and/or include the following:}

- Manufacturer and model of microscope

- Objective lens magnification, NA, and correction for aberration (e.g., 60x 1.4 NA Plan-Apochromat)

- Fluorescence filter set manufacturer and part number and/or the transmission and bandwidth (e.g., 490/30)

- Illumination light source (including wavelength for laser illumination)

- Camera manufacturer and model

- Software program(s) and version

- Manufacturer and model of other acquisition hardware, including confocal, filter wheels, focus motors, motorized stage, shutters, etc.

- Image acquisition settings including exposure times, gain, and binning

- Other acquisition parameters, including focus step size (for z-series), time between images (for time lapse), etc.

- Description of image processing routine used to create figures

- Description of segmentation and image analysis routine, and method of validation

intense focused illumination is used to photobleach fluorophores in a select region of a specimen. If the fluorescently labeled molecules are mobile, unbleached molecules will move into the bleached region while the bleached molecules move out. Recovery of the photobleached region over time can then be used to detect mobility/immobility, and to measure diffusion or association/ dissociation kinetics of the fluorescently labeled component (Snapp et al., 2003; Rabut and Ellenberg, 2005; Sprague and McNally, 2005).

As the specimen is illuminated to collect images of the recovery process, fluorophores will continue to photobleach at a rate dependent (in part) on the level of illumination. To get an accurate measure of recovery of the photobleached region, one must measure and correct for photobleaching that occurs during image acquisition. This can be done, for example, by measuring the fluorescence of an unbleached region in the same or a neighboring cell (Rabut and Ellenberg, 2005).

The accuracy of FRAP analyses is compromised by the fact that fluorophores can reversibly photobleach (Diaspro et al., 2006). It is therefore important to choose a fluorophore for FRAP experiments that is less likely to enter illumination-induced dark states that can spontaneously recover to the fluorescent state. EGFP, for example, is less likely to undergo reversible photobleaching than YFP variants. Reversible photobleaching can be detected by measuring the fluorescence intensity of entire cells after bleaching because it will cause the intensity to increase within a few seconds. The extent of the effect of reversibility of photobleaching on FRAP measurements depends on the rate of image acquisition, and can be controlled by collecting a number of pre-bleach images to reach a steady state of fluorophores in the dark state (Rabut and Ellenberg, 2005).

\section{Presenting quantitative} microscopy measurements Reporting error. No matter how careful you are when collecting images and making measurements, every quantitative analysis has a level of uncertainty that must be reported (Table II; Cumming et al., 2007; Wolf et al., 2007). Error is most commonly reported by stating or showing (as error bars) the standard deviation or standard error of the mean of the measured values. The appropriate way to report error depends on your data and the conclusions you would like to make. This journal recently published a thorough and user-friendly review on reporting error in quantitative measurements (Cumming et al., 2007). When performing arithmetic on multiple quantitative measurements that have independent sources of error (e.g., subtracting an average background intensity value $\mathrm{B} \pm \mathrm{b}$ from a measurement of signal intensity $S \pm s$ ), the error in the individual measurements should be propagated to the final value. A general formula for error propagation was derived by Wolf et al. (2007).

Writing the materials and methods. When publishing quantitative microscopy data, you should provide the reader with the information they need to judge whether you used the appropriate equipment and acquisition parameters for the experiment (Table III; Waters and Swedlow, 2007). Even with this information, it may be difficult for the reader to assess whether your system was optimized and operated to obtain the best possible SNR and resolution. Easy open access to raw image files and data used for published quantitative analyses will therefore be critical to the continued growth of the field of quantitative fluorescence microscopy (Moore et al., 2008).

\section{Further reading}

This review is an introduction to the issues surrounding accurate and precise quantitation of fluorescence microscopy digital images. A thorough appreciation of both the power and the limitations of quantitative microscopy is best obtained through careful attention to how these issues affect your own data. The interested reader is encouraged to learn more from the many excellent books, reviews, and quantitative analyses that are referenced throughout this review.

The author wishes to thank the faculty and students of the Analytical and Quantitative Light Microscopy course at the Marine Biological Laboratory (www.mbledu/education) for many illuminating discussions-especially Jason Swedlow, David Wolf, and John Murray; Jason Swedlow and Wendy Salmon for helpful comments and discussions on this manuscript; Cassandra Rogers for preparing bead slides used to acquire images for the figures; and Dan Bolton for patient support during the preparation of this manuscript.

\section{References}

Adams, M.C., W.C. Salmon, S.L. Gupton, C.S Cohan, T. Wittmann, N. Prigozhina, and C.M. Waterman-Storer. 2003. A high-speed multispectral spinning-disk confocal microscope system for fluorescent speckle microscopy of living cells. Methods. 29:29-41. 
Allan, V.J. 2000. Protein Localization by Fluorescence Microscopy: A Practical Approach. Oxford University, New York. 256 pp.

Art, J. 2006. Photon detectors for confocal microscopy. In Handbook of Biological Confocal Microscopy. J.B. Pawley, editor. SpringerVerlag New York, Inc., New York. 251-262.

Aubin, J.E. 1979. Autofluorescence of viable cultured mammalian cells. J. Histochem. Cytochem. 27:36-43.

Axelrod, D., N.L. Thompson, and T.P. Burghardt. 1983. Total internal inflection fluorescent microscopy. J. Microsc. 129:19-28.

Bolte, S., and F.P. Cordelieres. 2006. A guided tour into subcellular colocalization analysis in light microscopy. J. Microsc. 224:213-232.

Cardullo, R.A., and E.H. Hinchcliffe. 2007. Digital manipulation of brightfield and fluorescence images: noise reduction, contrast enhancement, and feature extraction. Methods Cell Biol. 81:285-314.

Chen, H., H.L. Puhl III, S.V. Koushik, S.S. Vogel, and S.R. Ikeda. 2006. Measurement of FRET efficiency and ratio of donor to acceptor concentration in living cells. Biophys. J. 91:L39-L41.

Churchman, L.S., Z. Okten, R.S. Rock, J.F. Dawson, and J.A. Spudich. 2005. Single molecule high-resolution colocalization of $\mathrm{Cy} 3$ and Cy5 attached to macromolecules measures intramolecular distances through time. Proc. Natl. Acad. Sci. USA. 102:1419-1423.

Cumming, G., F. Fidler, and D.L. Vaux. 2007. Error bars in experimental biology. J. Cell Biol. 177:7-11.

Day, R.N. 2005. Imaging protein behavior inside the living cell. Mol. Cell. Endocrinol. 230:1-6.

De Mey, J.R., P. Kessler, J. Dompierre, F.P. Cordelières, A. Dieterlen, J.L. Vonesch, J.B. Sibarita, and F.S. Kevin. 2008. Fast 4D microscopy. Methods Cell Biol. 85:83-112.

Diaspro, A., G. Chirico, C. Usai, P. Ramoino, and J. Dobrucki. 2006. Photobleaching. In Handbook of Biological Confocal Microscopy. J.B. Pawley, editor. SpringerVerlag New York, Inc., New York. 690-699.

Dorn, J.F., K. Jaqaman, D.R. Rines, G.S. Jelson, P.K. Sorger, and G. Danuser. 2005. Yeast kinetochore microtubule dynamics analyzed by high-resolution three-dimensional microscopy. Biophys. J. 89:2835-2854.

Dorn, J.F., G. Danuser, G. Yang, and F.S. Kevin. 2008. Computational processing and analysis of dynamic fluorescence image data. Methods Cell Biol. 85:497-538.

Egner, A., and S.W. Hell. 2006. Aberrations in confocal and multi-photon fluorescence microscopy induced by refractive index mismatch. In Handbook of Biological Confocal Microscopy. J.B. Pawley, editor. SpringerVerlag New York, Inc., New York. 404-412.

Evanko, D. 2009. Primer: fluorescence imaging under the diffraction limit. Nat. Methods. 6:19-20.

Giepmans, B.N., S.R. Adams, M.H. Ellisman, and R.Y. Tsien. 2006. The fluorescent toolbox for assessing protein location and function. Science. 312:217-224.

Goodwin, P.C. 2007. Evaluating optical aberration using fluorescent microspheres: methods, analysis, and corrective actions. Methods Cell Biol. 81:397-413.

Gordon, G.W., G. Berry, X.H. Liang, B. Levine, and B. Herman. 1998. Quantitative fluorescence resonance energy transfer measurements using fluorescence microscopy. Biophys. $J$. 74:2702-2713.

Hell, S.W., and E. Stelzer. 1995. Lens aberrations in confocal fluorescence microscopy.
In Handbook of Biological Confocal Microscopy. J.B. Pawley, editor. Springer-Verlag New York, Inc., New York. 347-353.

Hiraoka, Y., J.W. Sedat, and D.A. Agard. 1990 Determination of three-dimensional imaging properties of a light microscope system. Partial confocal behavior in epifluorescence microscopy. Biophys. J. 57:325-333.

Hoffman, D.B., C.G. Pearson, T.J. Yen, B.J. Howell, and E.D. Salmon. 2001. Microtubuledependent changes in assembly of microtubule motor proteins and mitotic spindle checkpoint proteins at PtK1 kinetochores. Mol. Biol. Cell. 12:1995-2009.

Huang, B., W. Wang, M. Bates, and X. Zhuang. 2008. Three-dimensional super-resolution imaging by stochastic optical reconstruction microscopy. Science. 319:810-813.

Inoue, S. 2006. Foundations of confocal scanned imaging in light microscopy. In Handbook of Biological Confocal Microscopy. J.B. Pawley, editor. Springer-Verlag New York, Inc., New York. 1-19.

Inoué, S., and K.R. Spring. 1997. Video Microscopy: The Fundamentals. Plenum Press, New York. 770 pp.

Jares-Erijman, E.A., and T.M. Jovin. 2003. FRET imaging. Nat. Biotechnol. 21:1387-1395.

Jares-Erijman, E.A., and T.M. Jovin. 2006. Imaging molecular interactions in living cells by FRET microscopy. Curr. Opin. Chem. Biol. 10:409-416.

Joglekar, A.P., D.C. Bouck, J.N. Molk, K.S. Bloom, and E.D. Salmon. 2006. Molecular architecture of a kinetochore-microtubule attachment site. Nat. Cell Biol. 8:581-585.

Joglekar, A.P., E.D. Salmon, K.S. Bloom, and F.S Kevin. 2008. Counting kinetochore protein numbers in budding yeast using genetically encoded fluorescent proteins. Methods Cell Biol. 85:127-151.

Johnson, I. 2006. Practical considerations in the selection and application of fluorescent probes. In Handbook of Biological Confocal Microscopy. J.B. Pawley, editor. SpringerVerlag New York, Inc., New York. 353-364.

Keller, H.E. 2006. Objective lenses for confoca microscopy. In Handbook of Biological Confocal Microscopy. J.B. Pawley, editor. Springer-Verlag New York, Inc., New York. 145-160.

Manley, S., J.M. Gillette, G.H. Patterson, H. Shroff, H.F. Hess, E. Betzig, and J. LippincottSchwartz. 2008. High-density mapping of single-molecule trajectories with photoactivated localization microscopy. Nat. Methods. 5:155-157.

Mason, D.W., and A.F. Williams. 1980. The kinetics of antibody binding to membrane antigens in solution and at the cell surface. Biochem. J. 187:1-20.

Melan, M.A., and G. Sluder. 1992. Redistribution and differential extraction of soluble proteins in permeabilized cultured cells. Implications for immunofluorescence microscopy. J. Cell Sci. 101:731-743.

Moomaw, B. 2007. Camera technologies for low light imaging: overview and relative advantages. Methods Cell Biol. 81:251-283.

Moore, J., C. Allan, J. Burel, B. Loranger, D. MacDonald, J. Monk, J.R. Swedlow, and F.S. Kevin. 2008. Open tools for storage and management of quantitative image data. Methods Cell Biol. 85:555-570.

Mortensen, K., and L.I. Larsson. 2001. Quantitative and qualitative immunofluorescence studies of neoplastic cells transfected with a construct encoding p53-EGFP. J. Histochem. Cytochem. 49:1363-1367.
Murray, J.M. 1998. Evaluating the performance of fluorescence microscopes. J. Microsc. 191:128-134.

Murray, J.M. 2005. Confocal microscopy, deconvolution and structured illumination methods. In Live Cell Imaging: A Laboratory Manual. R.D. Goldman and D.L. Spector, editors. Cold Spring Harbor Laboratory, Cold Spring Harbor, NY. 239-279.

Murray, J.M., P.L. Appleton, J.R. Swedlow, and J.C. Waters. 2007. Evaluating performance in three-dimensional fluorescence microscopy. J. Microsc. 228:390-405.

Nolte, A., J. Pawley, and L. Horing. 2006. Non-laser light sources for three-dimentional microscopy. In Handbook of Biological Confocal Microscopy. J.B. Pawley, editor. SpringerVerlag New York, Inc., New York. 126-144.

North, A.J. 2006. Seeing is believing? A beginners' guide to practical pitfalls in image acquisition. J. Cell Biol. 172:9-18.

Patterson, G.H., and D.W. Piston. 2000. Photobleaching in two-photon excitation microscopy. Biophys. J. 78:2159-2162.

Pawley, J. 2000. The 39 steps: a cautionary tale of quantitative 3-D fluorescence microscopy. Biotechniques. 28:884-886, 888

Pawley, J.B. 1994. Sources of noise in 3D microscopical data sets. In Three-Dimensional Confocal Microscopy: Volume Investigation of Biological Systems. J.K. Stevens, L.R. Mills, and J.E. Trogadis, editors. Academic Press, Inc., San Diego. 48-90.

Pawley, J.B. 2006a. Fundamental limits in confocal microscopy. In Handbook of Biological Confocal Microscopy. J.B. Pawley, editor. Springer-Verlag New York, Inc., New York. 20-42.

Pawley, J.B. 2006b. Handbook of Biological Confocal Microscopy. Springer-Verlag New York, Inc., New York. 985 pp.

Pawley, J.B. 2006c. Points, pixels, and gray levels: digitizing image data. In Handbook of Biological Confocal Microscopy. J.B. Pawley, editor. Springer-Verlag New York, Inc., New York. 59-79.

Periasamy, A., and R.N. Day. 2005. Molecular Imaging:FRETMicroscopy and Spectroscopy. Oxford University Press. 336 pp.

Ploem, J.S. 1999. Fluorescence microscopy. In Fluorescent and Luminescent Probes for Biological Activity. W.T. Mason, editor. Academic Press, London. 3-13.

Rabut, G., and J. Ellenberg. 2005. Photobleaching techniques to study mobility and molecular dynamics of proteins in live cells: FRAP, iFRAP, and FLIP. In Live Cell Imaging: A Laboratory Manual. R.D. Goldman and D.L. Spector, editors. Cold Spring Harbor Laboratory, Cold Spring Harbor, NY. 101-126.

Rasnik, I., T. French, K. Jacobson, and K. Berland. 2007. Electronic cameras for low-light microscopy. Methods Cell Biol. 81:219-249.

Rietdorf, J., and E.H.K. Stelzer. 2006. Special optical elements. In Handbook of Biological Confocal Microscopy. J.B. Pawley, editor. SpringerVerlag New York, Inc., New York. 43-58.

Robbins, M.S., and B.J. Hadwen. 2003. The noise performance of electron multiplying chargecoupled devices. IEEE Trans. Electron. Dev. 50:1227-1232.

Rocheleau, J.V., and D.W. Piston. 2003. Two-photon excitation microscopy for the study of living cells and tissues. Curr. Protoc. Cell Biol. Chapter 4:Unit 4.11.

Russ, J.C. 2007. The Image Processing Handbook. CRC/Taylor and Francis, Boca Raton, FL. 647 pp. 
Salmon, E.D., and J.C. Canman. 2001. Proper alignment and adjustment of the light microscope. Curr. Protoc. Cell Biol. Chapter 4:Unit 4.1.

Schaufele, F., I. Demarco, and R.N. Day. 2005 FRET imaging in the widefield microscope. In Molecular Imaging: FRET Microscopy and Spectroscopy. A. Periasamy and R.N. Day, editors. Oxford University Press. 72-94.

Sekar, R.B., and A. Periasamy. 2003. Fluorescence resonance energy transfer (FRET) microscopy imaging of live cell protein localizations. J. Cell Biol. 160:629-633.

Shaner, N.C., P.A. Steinbach, and R.Y. Tsien. 2005 A guide to choosing fluorescent proteins. Nat. Methods. 2:905-909.

Siegel, R.M., F.K. Chan, D.A. Zacharias, R Swofford, K.L. Holmes, R.Y. Tsien, and M.J. Lenardo. 2000. Measurement of molecular interactions in living cells by fluorescence resonance energy transfer between variants of the green fluorescent protein. Sci. STKE. 2000:PL1.

Snapp, E.L., N. Altan, and J. Lippincott-Schwartz. 2003. Measuring protein mobility by photobleaching GFP chimeras in living cells. Curr Protoc. Cell Biol. Chapter 21:Unit 21.1.

Sprague, B.L., and J.G. McNally. 2005. FRAP analysis of binding: proper and fitting. Trends Cell Biol. 15:84-91.

Spring, K.R. 2007. Cameras for digital microscopy. Methods Cell Biol. 81:171-186.

Straight, A.F. 2007. Fluorescence protein applications in microscopy. Methods Cell Biol. 81:93-113.

Stryer, L. 1978. Fluorescence energy transfer as a spectroscopic ruler. Annu. Rev. Biochem. 47:819-846.

Swedlow, J.R., K. Hu, P.D. Andrews, D.S. Roos, and J.M. Murray. 2002. Measuring tubulin content in Toxoplasma gondii: a comparison of laser-scanning confocal and wide-field fluorescence microscopy. Proc. Natl. Acad. Sci. USA. 99:2014-2019.

Tommre, D., and J.B. Pawley. 2006. Disk-scanning confocal microscopy. In Handbook of Biological Confocal Microscopy. J.B. Pawley, editor. Springer-Verlag New York, Inc., New York. 221-237.

Tsien, R.Y., L. Ernst, and A. Waggoner. 2006. Fluorophores for confocal microscopy: photophysics and photochemistry. In Handbook of Biological Confocal Microscopy. J.B. Pawley, editor. Springer-Verlag New York, Inc., New York. 338-348.

Vogel, S.S., C. Thaler, and S.V. Koushik. 2006. Fanciful FRET. Sci. STKE. 2006:re2.

Wallace, W., L.H. Schaefer, and J.R. Swedlow. 2001. A workingperson's guide to deconvolution in light microscopy. Biotechniques. 31:1076-1097.

Waters, J.C. 2007. Live-cell fluorescence imaging. Methods Cell Biol. 81:115-140.

Waters, J.C., and J.R. Swedlow. 2007. Interpreting fluorescence microscopy images and measurements. In Evaluating Techniques in Biochemical Research. D. Zuk, editor. Cell Press, Cambridge, MA. 37-42.

Wolf, D.E., C. Samarasekera, and J.R. Swedlow. 2007. Quantitative analysis of digital microscope images. Methods Cell Biol. 81:365-396.

Wu, J.Q., and T.D. Pollard. 2005. Counting cytokinesis proteins globally and locally in fission yeast. Science. 310:310-314.

Yildiz, A., and P.R. Selvin. 2005. Fluorescence imaging with one nanometer accuracy: application to molecular motors. Acc. Chem. Res. 38:574-582.

Zwier, J.M., G.J. Van Rooij, J.W. Hofstraat, and G.J. Brakenhoff. 2004. Image calibration in fluorescence microscopy. $J$. Microsc. 216:15-24. 